\title{
Physics with Leading Baryons at HERA
}

\author{
Armen Bunyatyan \\ Max-Planck-Institut für Kernphysik, \\ Saupfercheckweg 1, 69117 Heidelberg, Germany \\ and Yerevan Physics Institute, Armenia \\ E-mail: 'bunar@mail. desy.de',
}

\section{Representing the $\mathrm{H} 1$ and ZEUS Collaborations}

ABstRACT: Recent H1 and ZEUS results on leading baryon production are presented. The data cover a large kinematic range from photoproduction to deep inelastic scattering. The protons and neutrons are produced at very low angles and carry a large fraction of the incoming proton's energy. The mechanism of leading baryon production is studied. The factorization properties of leading baryon production are investigated.

\section{Introduction}

In this paper, we report the recent results of the $\mathrm{H} 1$ and ZEUS Collaborations on leading baryon production [1-4]. These are protons and neutrons which carry a large fraction of the energy of the target proton $\left(x_{L}>0.2\right)^{1}$ but are produced with small transverse momentum $\left(p_{T}<700 \mathrm{MeV}\right)$. Although the usual hadronisation mechanisms in strong interactions are expected to yield a fraction of these leading baryons, the exchange of colour singlet particles (e.g. pions) are also expected to contribute to their production.

The data cover a large kinematic range: from photoproduction PHP, $\left(Q^{2} \sim 0\right)$ to deep inelastic scattering (DIS), $\left(Q^{2}\right.$ up to $\left.250 \mathrm{GeV}^{2}\right)$. The studies include both semi-inclusive reactions $e p \rightarrow e^{\prime} N X$ as well as the production of jets with high transverse energies and vector mesons.

\section{Photoproduction with a Leading Proton}

The semi-inclusive reaction $e p \rightarrow e^{\prime} X p^{\prime}$ in photoproduction was investigated by the H1 experiment iin. The kinematic range of this measurement is restricted to values of the photon virtuality $Q^{2}<0.01 \mathrm{GeV}^{2}$ and the center-of-mass energy of the photon-proton

\footnotetext{
${ }^{1}$ The variable $x_{L}$ is defined as $x_{L} \equiv E_{p}^{\prime} / E_{p}$, where $E_{p}$ and $E_{p}^{\prime}$ are the energies of the target and scattered protons, respectively. In this paper the variables $x_{L}$ and $z$ refer to the same quantity.
} 
system $W$ between 60 and $250 \mathrm{GeV}$. The scattered proton energy is restricted to $0.66<$ $x_{L} \equiv E_{p}^{\prime} / E_{p}<0.88$ and the transverse momentum to $p_{T} \leq 200 \mathrm{MeV}$.

The cross section $d \sigma_{\gamma p \rightarrow X p^{\prime}}\left(W, x_{L}\right) / d x_{L}$ for three average values of $W$ and five values of $x_{L}$ is shown in Fig.1. It is observed that for all $x_{L}$ and $W$, the measured cross sections are compatible with each other within the experimental errors with the average cross section value of $d \sigma_{\gamma p \rightarrow X p^{\prime}}\left(W, x_{L}\right) / d x_{L}=8.05 \pm 0.06($ stat $) \pm 0.89($ syst $) \mu b$.

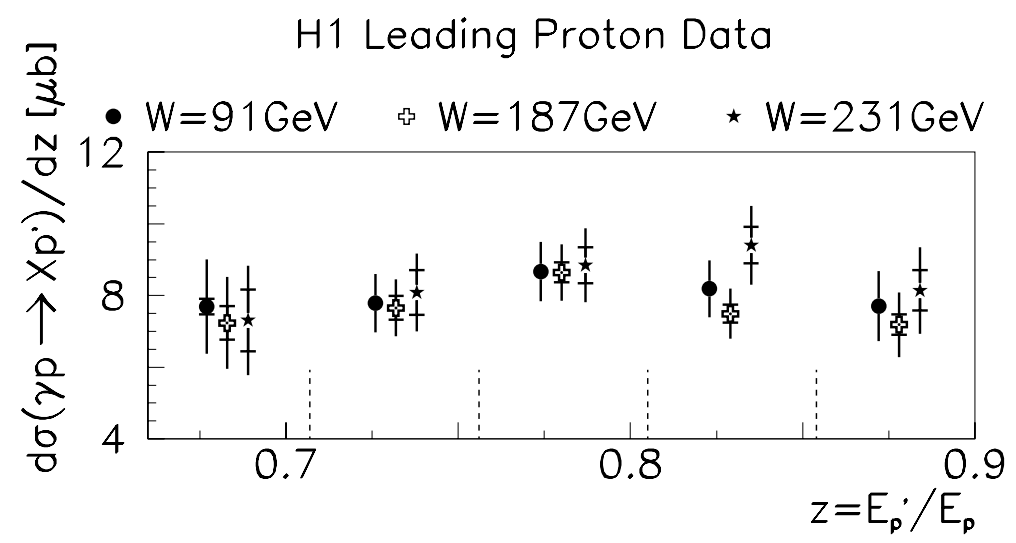

Figure 1: The cross section $d \sigma_{\gamma p \rightarrow X p^{\prime}} / d x_{L}$ as a function of $x_{L}$ for three values of $W$.

In Regge phenomenology, the $W$ and $x_{L}$ dependences of $d \sigma / d x_{L}$ yield information about the exchange mechanism contributing to leading proton production. Using the "Triple Regge" ansatz with the Mueller-Regge approach [īin], in this analysis, two single effective trajectories are considered which represent averages of the mixtures contributing to the reactions: $\alpha_{i}$ refers to the trajectory exchanged between the photon and the proton, and $\alpha_{k}$ refers to the trajectory describing the total cross section between $\alpha_{i}$ and the photon. A fit of this ansatz to the data gives:

$$
\left.\left.\alpha_{i}(0)=0.33 \pm 0.04(\text { stat. }) \pm 0.04 \text { (syst. }\right) \text { and } \quad \alpha_{k}(0)=0.99 \pm 0.01 \text { (stat. }\right) \pm 0.05 \text { (syst.). }
$$

The values of $\alpha(0)$ for the $\pi, \mathbb{R}$ and Pomeron trajectories are approximately $0,0.5$ and 1 , respectively. Hence, within the framework of the applied model, the exchange between the photon and the proton can be understood as a mixture of trajectories dominated by $\pi$ and $\mathbb{R}$ while the total cross section for the scattering between the mixture of Regge trajectories represented by $\alpha_{i}$ and the photon is dominated by Pomeron exchange.

\section{DIS with a Leading Proton and the Transition from PhP to DIS}

The ZEUS Collaboration has studied the semi-inclusive reaction $e p \rightarrow e^{\prime} X p^{\prime}$ in two kinematic ranges: at low $Q^{2}\left(0.1<Q^{2}<0.74 \mathrm{GeV}^{2}\right)$ and the DIS regime $\left(3<Q^{2}<\right.$ $\left.254 \mathrm{GeV}^{2}\right)$ [2]. The leading protons are measured in the range $0.6<x_{L}<0.97$ and $p_{T}<700 \mathrm{MeV}$. For each bin of $x$ (the Bjorken scaling variable) and $Q^{2}$, the quantity $r^{L P(3)}$ is defined as the ratio of the semi-inclusive cross section to the inclusive $(e p \rightarrow e X)$ cross section, differential in $x_{L}$, integrated over leading proton transverse momenta $p_{T}^{2}<0.5 \mathrm{GeV}^{2}$. The distributions of $r^{L P(3)}$ in different bins of $x, Q^{2}$ and $x_{L}$ are shown in Figure 2. 


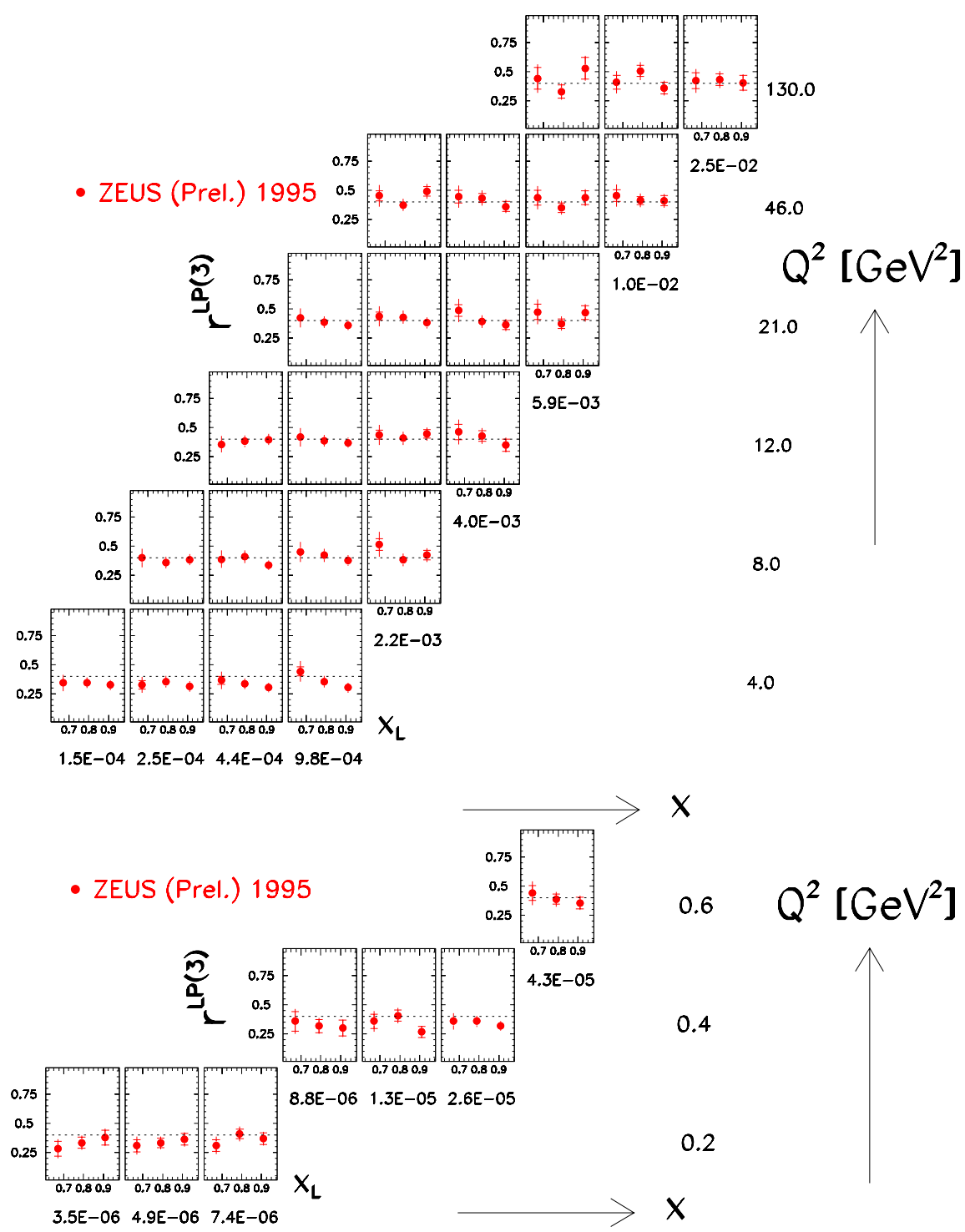

Figure 2: The ratio $r^{L P(3)}$ as a function of $x_{L}$ in bins of $x$ and $Q^{2}$, for protons with $p_{T}<700 \mathrm{MeV}$. The dashed lines $r^{L P(3)}=0.4$ are only to guide the eye.

The data show no significant $x$ or $x_{L}$ dependence, but indicate a slight rise with $Q^{2}$. This is investigated more closely by studying the ratio $r^{L P(3)}$ averaged over $x$ and $x_{L}$ as shown in Figure 3: the average ratio $\left\langle r^{L P(3)}\left(Q^{2}\right)\right\rangle$ grows by about $25 \%$ as $Q^{2}$ increases from 0.25 to $100 \mathrm{GeV}^{2}$.

The $Q^{2}$ dependence of leading proton production is further studied by the H1 Collaboration. The ratio of semi-inclusive cross sections for leading proton production to inclusive cross sections $f_{L P}\left(Q^{2}, W, x_{L}\right)$ is shown in Figure 4, separately for each $x_{L}(\equiv z)$. The fraction of leading proton events increases with $Q^{2}$ from $5 \%$ per unit of $x_{L}$ in photoproduction $\left(Q^{2} \sim 0\right)$ to about $10 \%$ at $Q^{2} \sim 10 \mathrm{GeV}^{2}$ at all $x_{L}$ and $W$.

The observed rise of $f_{L P}$ and $\left\langle r^{L P(3)}\right\rangle$ with $Q^{2}$ indicates a breaking of factorization of the proton and photon vertices. Absorptive corrections, corresponding to multiple Reggeon 


\section{ZEUS}

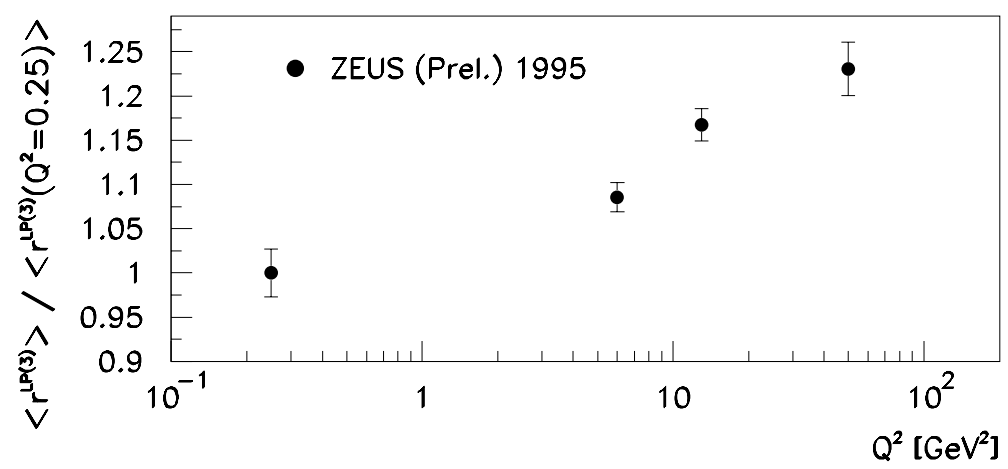

Figure 3: The average ratio $\left\langle r^{L P(3)}\right\rangle$ as a function of $Q^{2}$, for protons with $p_{T}<700 \mathrm{MeV}$, normalized to the value at $Q^{2}=0.25 \mathrm{GeV}^{2}$.

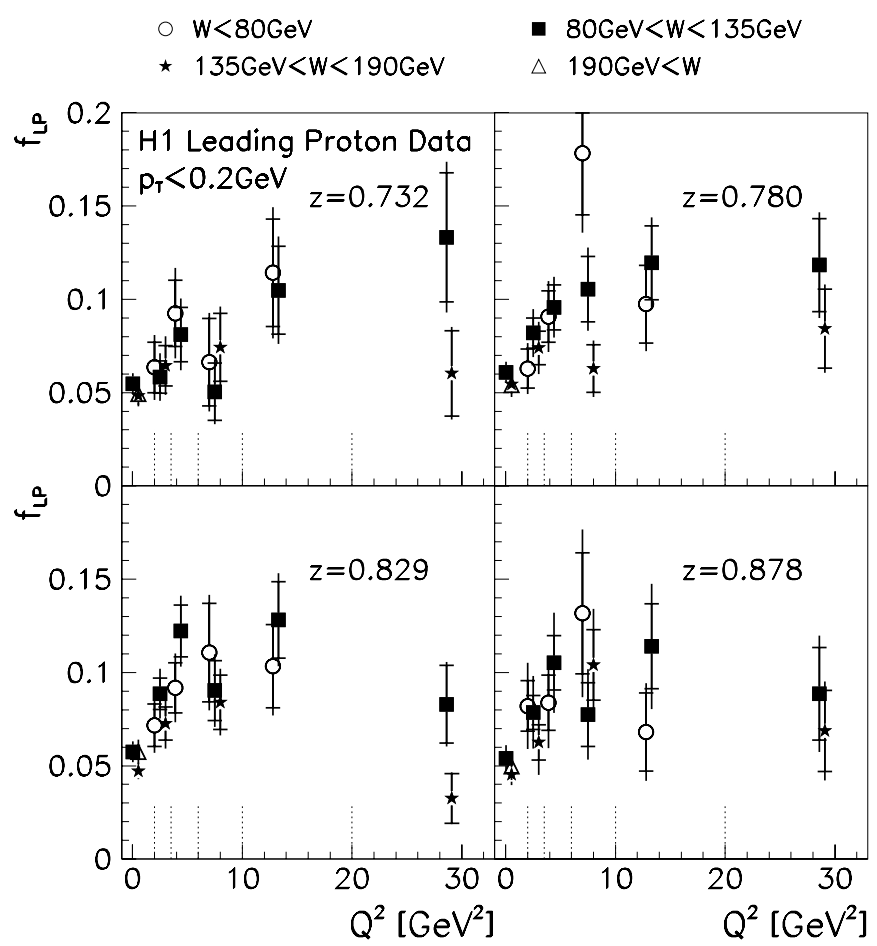

Figure 4: The ratio $f_{L P}$ per unit $z \equiv x_{L}$ of the leading proton to the total cross section for four values of $z$ in four intervals of $\mathrm{W}$ as a function of $Q^{2}$.

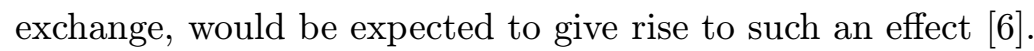

\section{Dijet production with Leading Neutrons}

Dijet production was studied in events which contain, in the final state, a leading neutron with energy $E_{n}>400 \mathrm{GeV}$ and polar angle $\theta_{n}<0.8 \mathrm{mrad}\left[\begin{array}{l}3 \\ 3\end{array}\right]$.

Figure 5(a) shows the uncorrected neutron energy distribution for events containing 

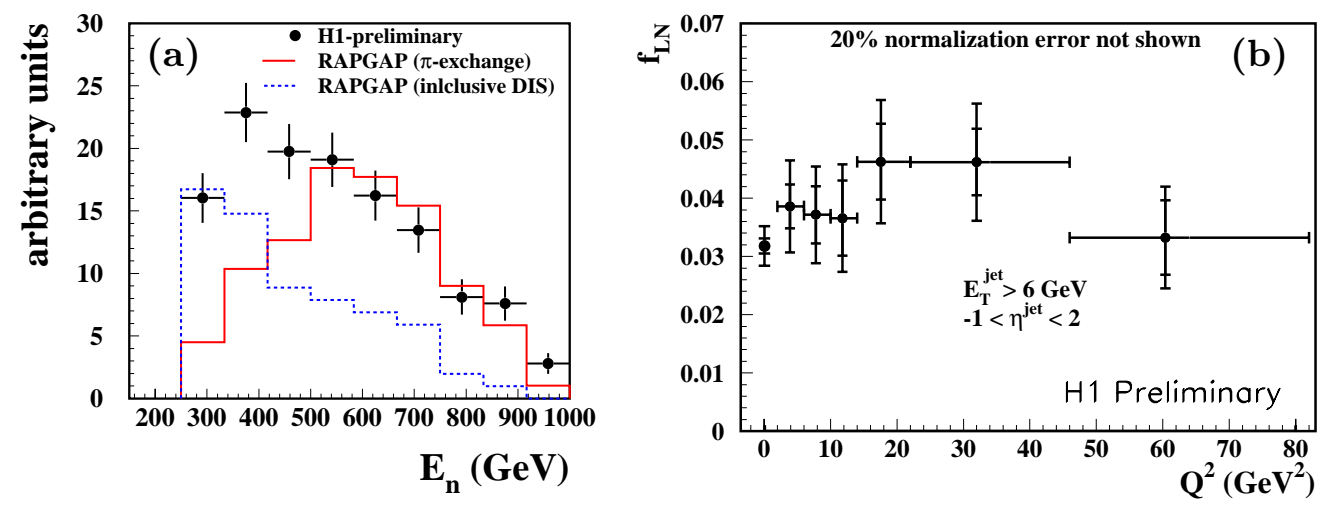

Figure 5: (a) The uncorrected distribution of leading neutron energy $E_{n}$ in dijet events for DIS data $\left(2<Q^{2}<80 \mathrm{GeV}^{2}\right)$ compared with two predictions using the RAPGAP Monte Carlo generator. (b) The fraction of dijet events with a leading neutron as a function of $Q^{2}$. The first point corresponds to photoproduction $\left(Q^{2} \sim 0\right)$.

two jets with transverse energies $E_{T}^{j e t}>6 \mathrm{GeV}$. The data are compared with two simulations using the RAPGAP Monte Carlo generator [i]. The inclusive DIS simulation contains no colour singlet exchange and describes the data only in the low $E_{n}$ region where significant leading neutron production is expected from hadronisation. On the other hand, the model based on pion exchange gives a good description for $E_{n} \gtrsim 500 \mathrm{GeV}\left(x_{L} \gtrsim 0.55\right)$, indicating that colour singlet exchange is the dominant production mechanism in this region.

The factorization property of dijet events with leading neutrons has been studied by measuring the fraction of events with a leading neutron in the inclusive dijet sample as a function of $Q^{2}$. The resulting distribution is shown in Figure 5(b). The observed fraction of dijet events with a leading neutron shows no significant dependence on $Q^{2}$, in agreement with the hypothesis that the proton-pion and pion-photon vertices are independent.

\section{Proton-dissociative photoproduction of $\rho$-mesons at large momentum transfer squared with a leading baryon}

For a further test of the factorization of leading nucleon production, the ZEUS Collaboration has investigated the proton-dissociative $\rho$-meson photoproduction process with large momentum transfer squared between the photon and the $\rho$-meson $\left(|t|>1.2 \mathrm{GeV}^{2}\right)\left[\begin{array}{l}{[\overline{4}} \\ 0\end{array}\right]$. The process studied is $\gamma p \rightarrow \rho Y$, where $Y$ is the proton dissociative system which contains a proton or a neutron with a large fraction of the incoming proton's beam energy, $x_{L}>0.2$.

The yield of leading nucleons in proton-dissociative $\rho$-meson photoproduction is compared with those in the fully inclusive DIS process. Figures 6(a) and 6(b) show the fractions of events that contain a leading proton with $p_{T}^{2}<0.5 \mathrm{GeV}^{2}$ for the full DIS sample and for high- $|t|$ proton dissociative $\rho$ meson photoproduction respectively.

The ratio of these two ratios is shown in Figure 6(c), giving a comparison of both rate and shape for the two processes. The ratio is almost constant with $x_{L}$ and close to unity. Moreover, it is observed that the leading nucleons in these two processes have similar angular distributions (not shown). 

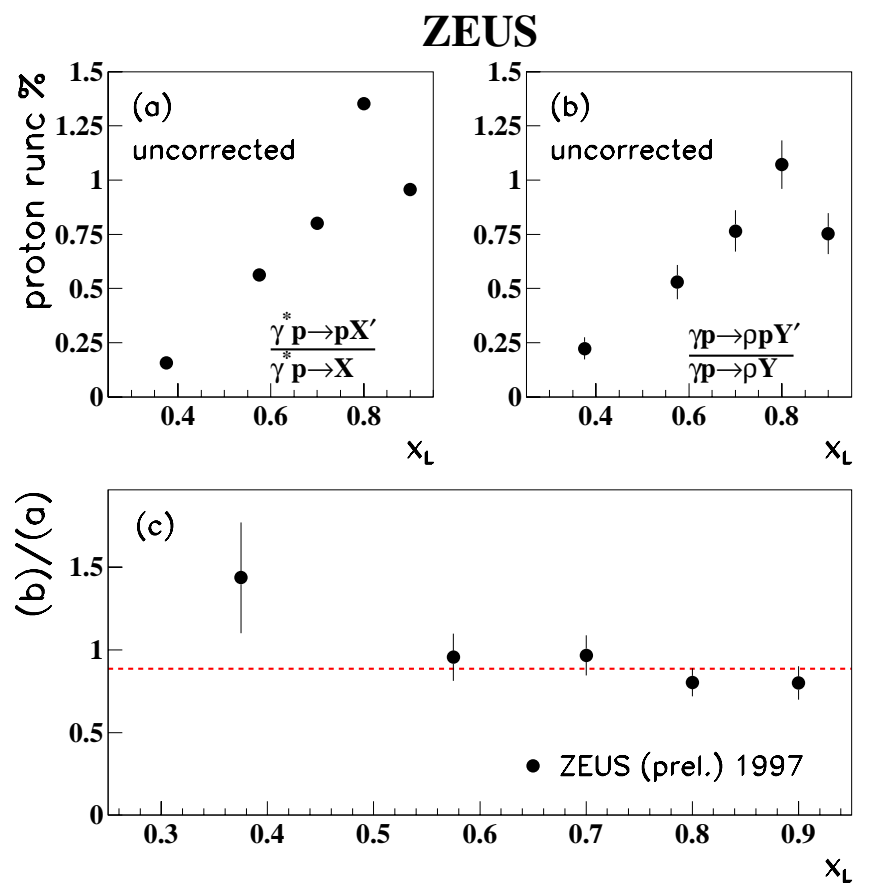

Figure 6: The rate of protons with $p_{T}^{2}<0.5 \mathrm{GeV}^{2}$ in (a) DIS $Q^{2}>24 \mathrm{GeV}^{2}$, and in (b) photoproduced proton dissociation $\rho$ events, $|t|>1.2 \mathrm{GeV}^{2}$, as a function of $x_{L}$. (c) The ratio of the two rates as a function of $x_{L}$. The dotted line shows the average value of double ratio for protons with $0.25<x_{L}<0.95$.

Summarizing, several tests of vertex factorization have been performed. In general, the data support the hypothesis that the dependence of the cross section on the nucleon variables is independent of the particle production at the photon vertex, but there is some indication of a factorization breaking.

\section{References}

[1] H1 Collaboration, "Photoproduction with a Leading Proton at HERA",DESY 01-062, May 2001.

[2] ZEUS Collaboration, "Leading proton production in $e^{+} p$ collisions at HERA", Abstract 567, International Europhysics Conference on High Energy Physics, Budapest, 2001.

[3] H1 Collaboration, "Measurement of dijet cross sections with leading neutrons at HERA", Abstract 811, International Europhysics Conference on High Energy Physics, Budapest, 2001.

[4] ZEUS Collaboration, "Proton-dissociative photoproduction of vector mesons at large momentum transfer with a leading nucleon at HERA", Abstract 652, International Europhysics Conference on High Energy Physics, Budapest, 2001.

[5] A.H.Mueller, Phys. Rev. D2, (1970) 2963.

[6] N.N.Nikolaev, J.Speth and B.G.Zakharov, [hep-ph/9708290].

[7] H. Jung, Comp. Phys. Comm. 86, (1995) 147. 